\title{
An Energy Efficient Knee Locking Mechanism for a Dynamically Walking Robot
}

\author{
Gijs van Oort*, Raffaella Carloni*, Dian J. Borgerink** and Stefano Stramigioli*
}

\begin{abstract}
In this work, we present the design and the implementation of an innovative knee locking mechanism for a dynamically walking robot. The mechanism consists of a fourbar linkage that realizes a mechanical singularity for locking the knee when the leg is in the extended position. Once extended, the knee remains locked without energy consumption, while unlocking it only costs a small amount of energy. Tests showed that the robot walks robustly and that the energy consumption of the new system is low.
\end{abstract}

\section{INTRODUCTION}

The energy efficient bipedal walking robot Dribbel (Fig. 1), developed at the Control Engineering group of the University of Twente, has been built in order to demonstrate the potential dynamic walking [1]. Dribbel has a mass of $16.1 \mathrm{~kg}$ and its leg length is $0.95 \mathrm{~m}$. Instead of using heavily geared motors, the knees of Dribbel have no motor at all. Nevertheless, the robot is able to produce a stable dynamic gait. In order to keep the legs extended during stance phase, the knees of Dribbel are equipped with a locking system.

The original knee lock design (Fig. 2) used electromagnets to keep the leg stretched. With these knee locks Dribbel was able to walk, but sometimes it stumbled, due to the fact that the mechanism failed to fix the leg. When, for example, the lower leg swung too fast, the electromagnets could not absorb all kinetic energy, resulting in a rebound of the leg.

Another problem of the knee locking mechanism was that the locking force was limited. The ankle push-off mechanism made by Franken [2] (a bi-articular one, i.e., the force runs over the knee and the ankle) produces large knee torques, sometimes resulting in a spontaneous unlocking of the knee.

A third disadvantage of the previous knee lock was that the mechanism consumed a significant amount (55\%) of the total energy of the robot (the electromagnets were switched on for $77 \%$ of the time and consumed 17 of the total $31 \mathrm{~W}$ used by Dribbel).

We have conducted a study on different knee locking mechanisms [3]. Based on that, an innovative design was made [4], which uses a mechanical singularity to lock the knee - effortless. The advantages of the new mechanism over the previous electromagnet version are:

- Chances of rebound of the lower leg are much smaller,

This work has been funded by the European Commission's Seventh Framework Programme as part of the project VIACTORS under grant no. 231554.

* \{g.vanoort,r.carloni,s.stramigioli $\} @$ utwente.nl, Department of Electrical Engineering, Faculty of Electrical Engineering, Mathematics and Computer Science, University of Twente, 7500 AE Enschede, The Netherlands.

**d.j.borgerink@student.utwente.nl, Deptartment of Advanced Technology, Faculty of Science and Technology, University of Twente, 7500 AE Enschede, The Netherlands.

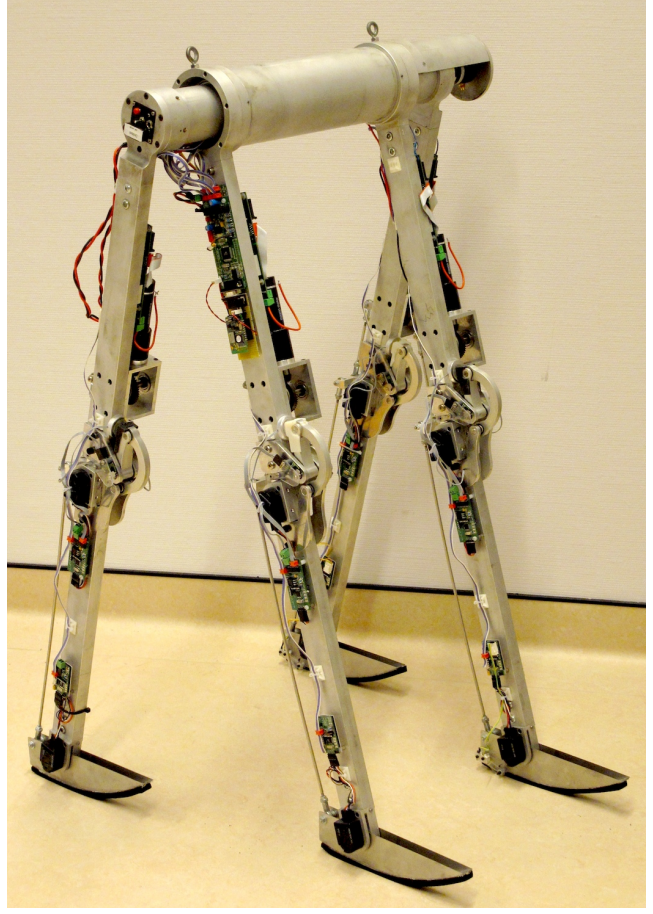

Fig. 1. The dynamically walking robot Dribbel.

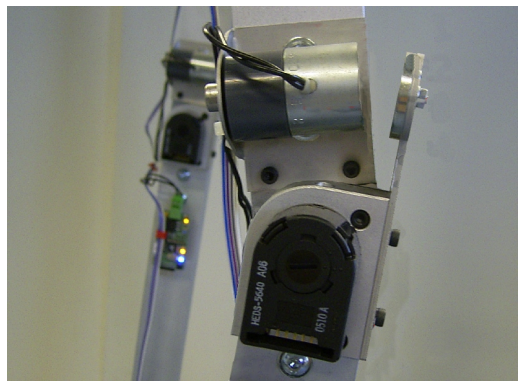

Fig. 2. The old knee locking mechanism of Dribbel: An electomagnet pulling an iron disk (top right) towards itself. The magnet had to be turned on permanently in order to keep the knee locked.

- The mechanism uses only a small amount of energy to keep locked,

- The locking force is independent on the energy consumption of the mechanism; it is only dependent on the mechanical strength of the mechanism.

All dynamically walking robots with knees suffer from the problem of needing a knee mechanism. The first kneed walker, by McGeer, did not have any locking mechanism at all; it only had a de-bouncing mechanism and an endstop [5]. 
The 2D biped Mike, by Wisse, used a mechanical latch and a solenoid for unlocking [6]. The 3D walker by Collins has an electromagnetic release system for a movable latch [7]. At MIT, Baines developed a knee mechanism with a DC motor for actuation and an electromagnetic clutch for uncoupling the actuator from the knee [8]. This mechanism was later improved by Reynolds [9] by using bevel gears. Trifonov et al. tried a totally passive locking system with permanent magnets, but this could not be tuned well enough. Later they developed a mechanism with a locking hook [10].

In the world of prosthetics there are many patents on knee mechanisms. Many describe four-bar linkages that create a virtual rotation point outside the mechanism, or a virtual rotation point that moves during the flexion of the knee [11], [12], [13]. Generally, these systems are constructed such that exerting a pressure on the knee (i.e., leaning on the extended leg) helps locking the system. Another four-bar mechanism is found in [14], where the mechanism is constructed such that it acts as a pivot lock. A knee system with a dedicated locking hook is described in [15]. A locking system using a mechanical singularity is described in [16], although it has noting to do with prostheses or robots (it is used for printing or varnishing machines).

The paper is organized as follows. In Sec. II we state the design requirements for our new knee lock. Then, in Sec. III we present the new knee locking system and discuss some of the features. A few tests and measurements done on the new actuator itself and on Dribbel are described in Sec. IV. Finally, before concluding, we show in Sec. V how mechanical play (backlash) appears to be advantageous.

\section{DESIGN REQUIREMENTS}

In this paper, the knee angle, $\varphi$, is defined as the angle between upper and lower leg, where $\varphi=0$ corresponds to a fully extended (i.e., straight, not bent) leg, and a bent knee will give a positive value for $\varphi$.

In order to be suitable for our research on dynamically walking robots, the new knee locking mechanism has to meet the following requirements:

- The knee locking mechanism should be able to lock the knee when the leg becomes fully extended, preventing it from flexing again,

- When the knee is flexed (during swing phase), the mechanism's influence on the dynamics of the leg should be minimal,

- The mechanism should be energy efficient, lightweight and robust against falling.

The maximum amount of flexing torque expected is due to the push-off. From measurements on Dribbel, it is deduced the maximum flexing torque is in the range of 3-5 Nm. The lock mechanism should be able to withstand this. As no movement at all is needed during this torque, it would be logical to use a high transmission ratio $n$ between the actuator used and the knee. Clearly, this is only needed when the leg is straight $(\varphi=0)$.
Dribbel has been designed to have a passive-dynamic walking gait, which means that the passive dynamics of the system dominate the movements (as opposed to actuators dominating the movement). Hence, the influence of the new knee lock on the dynamics should be minimized. Minimizing the mass of the mechanism is therefore important, as well as minimizing the added moment of inertia. It is desirable to have only a small transmission ratio $n$ between the actuator and the knee, since a large transmission ratio would increase the apparent inertia of the actuator by $n^{2}$. This is important especially during the swing phase, when there is most movement of the leg and the leg is bent $(\varphi>0)$.

It is clear that we have contradicting requirements: on one hand the transmission ratio between the actuator and knee should be high in order to achieve a large locking torque with a small actuator, on the other hand it should be low in order to minimize the added inertia. Fortunately, these two requirements are needed in two different regions of the knee angle: large transmission ratio when the leg is extended $(\varphi=0)$, and small transmission ratio when the leg is flexed $(\varphi>0)$. Therefore, a system that links the transmission ratio and the knee angle may give us the best of both worlds.

\section{THE NEW KNEE LOCKING MECHANISM: THE 'BEUGEL'}

Figs. 3 and 4 show the new knee locking mechanism. The mechanism (which is basically a four-linkages mechanism) consists of two rods, called the motor arm and the connection rod, connecting the upper and lower leg through rotational joints $J_{u}$ (on upper leg), $J_{l}$ (on lower leg) and $J_{m}$ (on motor arm). The fourth joint is the knee joint $J_{k}$ itself. A voice coil actuator (VCA) [17] from a hard disk can exert a torque $\tau_{\mathrm{VCA}}$ around $J_{u}$ onto the motor arm. The angle of the motor arm is called $\alpha$. At the end of the motor arm a suction cup with a small hole is mounted that can contact the end stop on the upper leg. There are also end stops on the lower leg which limit its range to $0 \leq \varphi \leq \pi / 2 \mathrm{rad}$.

\section{A. Four-linkages mechanism}

There is a fixed relation between the motor arm angle and the lower leg angle, as shown in Fig. 5. This relation is dependent on the exact geometry of the mechanism (Fig. 4b) and was found using numerical optimization [3]. The transmission ratio $n(\varphi)$ between motor arm angle (on which the actuator is mounted) and knee angle is the slope $n=\mathrm{d} \alpha / \mathrm{d} \varphi$ and is shown in Fig. 6. Indeed, we have the best of both worlds: a very large (even $\infty$ ) transmission ratio near $\varphi=0$ and a small transmission ratio for a bent knee. The torque on the lower leg (around $J_{l}$ ) that the system can generate is $\tau_{l}=n(\varphi) \cdot \tau_{\mathrm{VCA}}$.

Fig. 4b shows the system in its mechanical singularity. The transmission ratio is infinity, implying that the lower leg cannot rotate at all and, irrespective of the (external) torque on the knee, the motor arm can be kept in place with minimum energy consumption. This is an unstable equilibrium. 


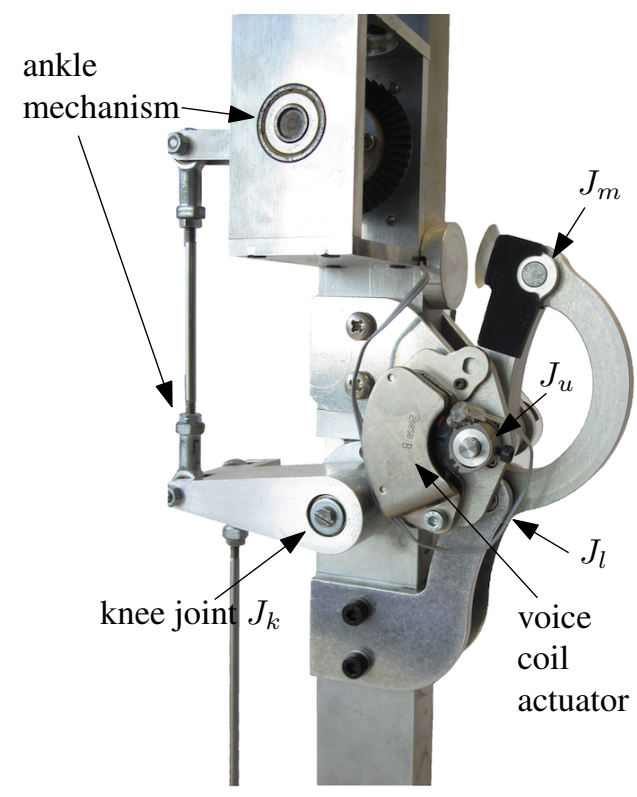

Fig. 3. The new knee locking mechanism.

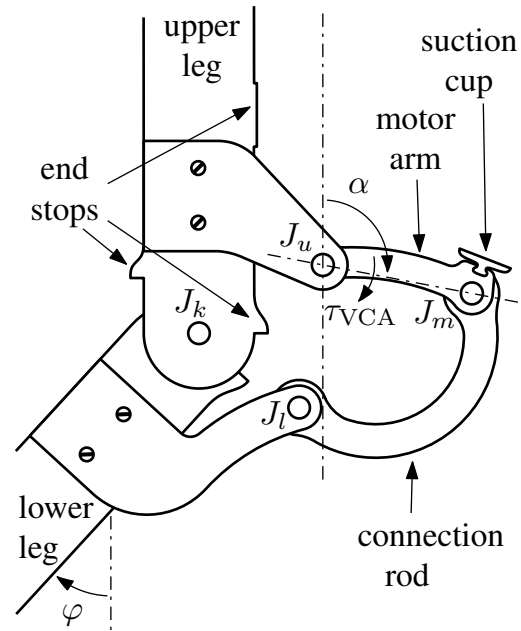

a

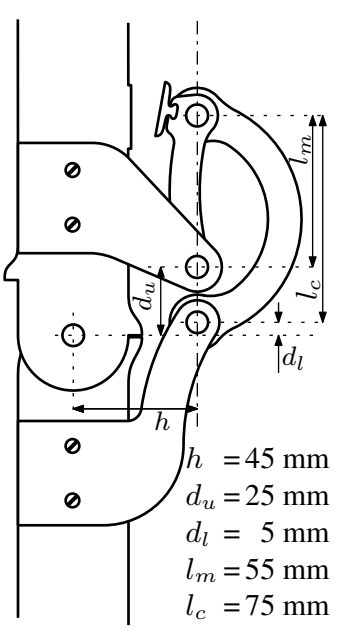

b
Fig. 4. The new knee locking mechanism. a) Leg in flexed position; b) System in singularity (joints $J_{m}, J_{u}$ and $J_{k}$ lie on one line).

The motor arm can go through the singularity, such that $\alpha<0$. This way, the suction cup on the motor arm can contact the end stop on the upper leg. The lower leg is then flexed a minimal small amount. If an (external) flexing force acts on the knee, it will cause the motor arm to be pressed harder against the end stop. Hence the knee cannot be flexed any further, i.e., the knee is locked.

Although using the mechanical singularity of a four-bar linkage has, to our best knowledge, never been used to make passive knee locking systems for robots or prostheses, the idea to use mechanical singularities to lock things is not new, and it is frequently used. Our direct inspiration came from our local beer brewery Grolsch, that ships their beer in so-

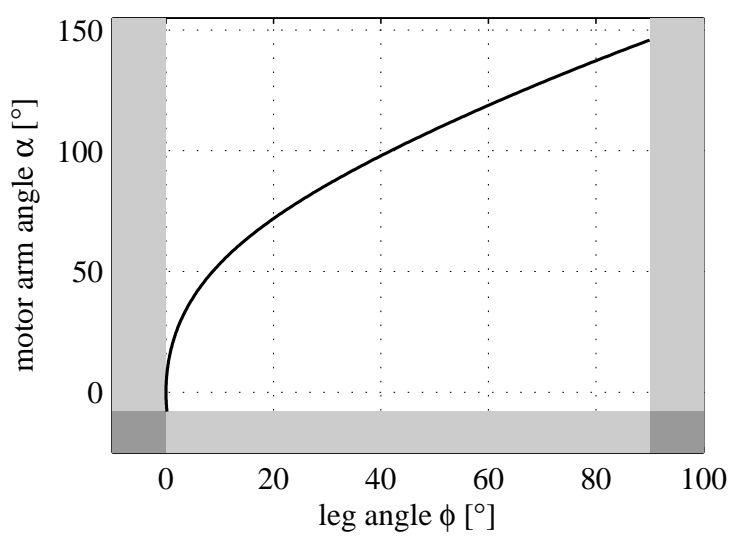

Fig. 5. The relation between motor arm angle $\alpha$ and leg angle $\varphi$. The gray areas are areas where the system cannot go because of the end stops. The areas at the side are due to the end stops at the knee joint; the area at the bottom is due to the suction cup end stop.

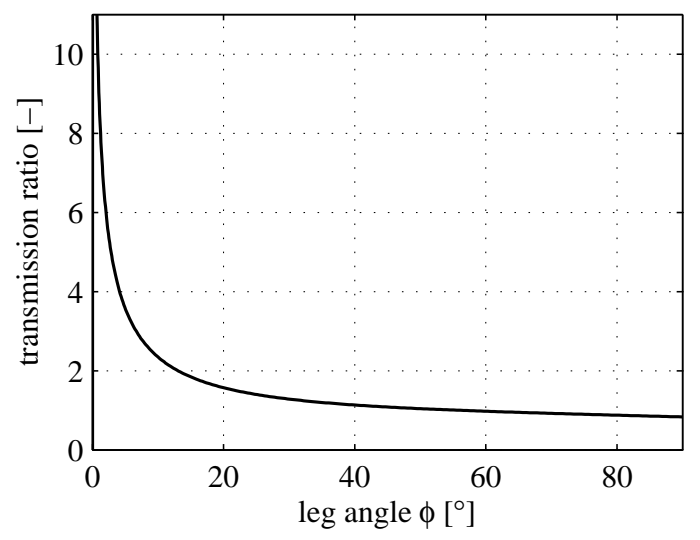

Fig. 6. The transmission ratio between motor arm angle (where the actuator is situated) and leg angle. For very small leg angles (near leg extension) the transmission ratio is high - a large torque can be exerted. When the leg is flexed, the transmission ratio quickly decreases, allowing free leg swing. After the motor arm has past its singularity, the transmission ratio becomes negative. This is out of the the scale of the graph.

called 'swing top bottles", (Grolsch Beugels in Dutch, hence the name for our device). Another example is the mechanism used to open and close small windows. These two examples are shown in Fig. 7. For comparison, we put the names used in this paper on the equivalent parts in the mechanisms.

\section{B. Suction cup}

Consider the event of locking the knee. When, during the end of the swing phase, the leg extends with velocity $\dot{\varphi}$, the motor arm will have an angular velocity $\dot{\alpha}=n \dot{\varphi}$. With the leg almost extended, $n$ is very large and so will $\dot{\alpha}$ be. Because of its own inertia (which is small but not zero), the motor arm will still have a substantial angular momentum when hitting the end stop. It is essential that the motor arm does not bounce back too far, otherwise the system may become unlocked again. This is where the suction cup comes into play. A suction cup with a small hole in it is known to be

\footnotetext{
${ }^{1}$ The swing top bottle was originally invented in 1875 by C. De Quillfeldt [18].
} 


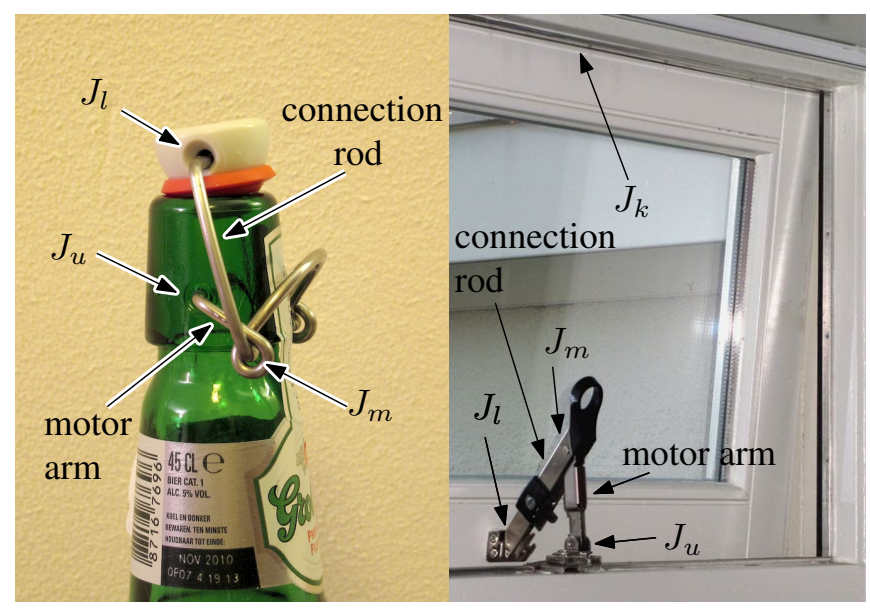

Fig. 7. Examples of other four-bar mechanisms using the mechanical singularity for locking. a) The Grolsch swing top bottle. The equivalent of $J_{k}$ is absent; the mechanism only keeps $J_{l}$ pulled towards $J_{u}$. b) A window opener.

able to absorb a lot of kinetic energy during collision, while having a minimum effect during non-collision. They were first used in McGeer's planar walking model with knees [5]. The working principle is as follows. At collision, the suction cup is pressed firmly against the end stop, squeezing out all air between the suction cup and end stop, generating a vacuum. The environmental air pressure then presses the suction cup against the end stop. A conventional (and ideal) suction cup would stick to the end stop forever, preventing the lock from opening during the next step. The small hole in our suction cups however, allows air to slowly leak into the space between the suction cup and end stop again, and after a while (typically a few tens of a second) the suction cup can be moved around freely again.

\section{Actuator}

The stroke of a typical voice coil motor from a hard disk is quite small (the one we used was $45^{\circ}$ ); from Fig. 5 it can be seen that the stroke of the VCA should be at least $155^{\circ}$ (as it it fixed to the motor arm, it should be capable of rotating through the full range of $\alpha$ ). Therefore, one of the end stops of the actuator was removed so that the voice coil can leave the magnet housing of the VCA when the lower leg flexes. Outside the magnet housing, the VCA cannot generate any torque. However, this is not a problem, because the knee should not be actuated when the leg is flexed anyway.

\section{Electronics}

Dribbel's electronics are modular. It has one master module, the main controller board, containing an ATMega128 microcontroller and 13 slave boards: a hip actuator module, four ankle actuator modules, four knee joint modules and four ankle sensor modules. Each slave module is equipped with an ATMega8 microcontroller to perform local tasks such as interfacing with sensors, doing elementary calculations and passing its status or other data to the main controller. Communication is done using a TWI field bus.

\begin{tabular}{lll}
\hline State & Motor arm region & Actuator action \\
\hline \multirow{3}{*}{ Lock } & Unlocked, out of VCA range & None \\
& Unlocked, within VCA range & Negative direction \\
Locked & None \\
\hline \multirow{3}{*}{ Unlock } & Unlocked, out of VCA range & None \\
& Unlocked, within VCA range & Positive direction \\
& & Positive direction \\
\hline
\end{tabular}

TABLE I

CONTROL STRATEGY FOR THE MECHANISM. FOR EACH OF THE TWO STATES THE SYSTEM CAN BE IN, THE ACTUATOR ACTION IS SHOWN FOR THE DIFFERENT REGIONS OF THE MOTOR ARM.

The original knee joint modules were designed to simply turn on or off the electromagnet through a power transistor. The modules were adapted to the new knee lock by adding a full H-bridge. This allows both positive and negative actuation of the VCA's, as well as PWM regulation.

\section{E. Sensors}

In order to properly control the knee mechanism, the angle of the motor arm needs to be known. An encoder was already available on Dribbel to measure the knee angle, but this cannot be used to calculate the angle of the motor arm because the relation $\varphi=\varphi(\alpha)$ is non-injective (i.e., for some knee angles $\varphi$ there are multiple possible values for $\alpha$ ). It would be possible to move the encoder from the knee to the motor arm and then calculate the knee angle from the motor arm angle, but in that position it is more vulnerable when the robot falls.

Therefore, a new, simple sensor was added to get information about the angle of the motor arm. A small study showed that for the type of control conducted at this moment, it sufficed to divide the total stroke of the motor arm into three regions and only know in which region the motor arm is. The regions are:

- Unlocked, out of actuation range: $\alpha \geq 31^{\circ}$,

- Unlocked, within actuation range: $0^{\circ} \leq \alpha<31^{\circ}$,

- Locked: $\alpha<0^{\circ}$.

A custom sensor was made using two simple optical sensors. Two small non-transparent plates connected to the motor arm move through these sensors to indicate in which region the motor arm is. The plates can easily be adjusted to fine-tune the system.

\section{F. Control}

The control that is currently implemented in the new knee mechanism is straightforward. The knee locking system has two states: lock (try to lock the knee and keep it locked) and unlock (similarly defined). The main controller determines which state each knee should be in and sends the appropriate signals to the knee modules.

Depending on the state and the region the motor arm is in, a controller action (Table I) is selected. Note that the controller always steers the system towards a no-power region. Therefore, the current consumption is only large 


\begin{tabular}{lrl}
\hline Description & \multicolumn{2}{c}{ Value } \\
\hline Average step length & 0.29 & $\mathrm{~m}$ \\
Average step time & 1.0 & $\mathrm{~s}$ \\
Average velocity & 0.29 & $\mathrm{~m} / \mathrm{s}$ \\
Power consumption & 27.7 & $\mathrm{~W}$ \\
Specific cost of transport & 0.60 & - \\
\hline
\end{tabular}

TABLE II

SPECIFICATIONS OF THE GAIT OF DRIBBEL.

during the state transitions, not when in a state for a longer time.

\section{TESTS AND MEASUREMENTS ON DRIBBEL}

A. Power consumption of the knee mechanisms during normal gait

When fully powered $(U=12 \mathrm{~V})$, the VCA of the knee mechanism takes approximately $I=0.39 \mathrm{~A}$, which results in a power consumption of $P_{\mathrm{VCA}}=U \cdot I=4.7 \mathrm{~W}$ for each actuator. This is a significant amount, but it should be remembered that most of the time the actuators don't have to work at full power. During a normal walk, we measured the average actuation time for each actuator to be approximately $10 \%$ of the gait cycle, which implies that the four knee locks together consume approximately $1.9 \mathrm{~W}$.

\section{B. Total power consumption, specific cost of transport}

The specific cost of transport $C_{e t}$ is a non-dimensional indicator of the energy-efficiency of transportation. It equals the amount of (electrical) energy needed to transport a unit weight over a unit distance:

$$
c_{e t}=\frac{E}{m \cdot g \cdot d}=\frac{P_{\mathrm{avg}}}{m \cdot g \cdot v_{\mathrm{avg}}}
$$

where $E$ is the energy used, $m$ the mass of the system, $g$ the earth's gravitational acceleration, $d$ the distance traveled, $P_{\text {avg }}$ the average power consumed and $v_{\text {avg }}$ the average velocity.

In order to obtain the average power consumption, we made Dribbel walk and recorded the power consumption as a function of time. Over four typical, consecutive steps, we measured an average power consumption of $27.7 \mathrm{~W}$. The hip actuator was controlled by a simple PD controller that instantaneously switches the setpoint for the inter-leg angle when the front foot touches the ground (similar to [19], but with the difference that we used very low controller gains such that the swing phase more or less resembles natural swinging motion).

Dribbel's velocity was measured by making it walk 14 consecutive steps, covering a distance of $4.0 \mathrm{~m}$. This took $14.0 \mathrm{~s}$, giving an average velocity of $v_{\mathrm{avg}}=0.29 \mathrm{~m} / \mathrm{s}$. Together with the mass of Dribbel being $16.1 \mathrm{~kg}$, we find a specific cost of transport of $c_{e t}=0.60$. Table II shows some important specifications of Dribbel.

\section{HOW MECHANICAL PLAY IS -FOR ONCE- OUR FRIEND}

When we first implemented the new knee mechanisms on Dribbel, the system did not work entirely as expected. Often, the motor arm did not pass its singularity during kneestrike, and when it did, it did not go to the end stop but was pushed back towards the singularity (which is unwanted because a small disturbance on the motor arm could cause it to move into the unlocked region again). The behavior was also heavily non-reproducible: one day it could work very well; the other day it could fail all the time. We identified the source of the problems: the singularity itself.

For the motor arm not passing the singularity during knee-strike, we found two opposing causes. The system was designed such that it could be fine-tuned easily (the positions of $J_{l}$ and $J_{u}$ can be changed a few $\mathrm{mm}$ by loosening and re-tightening a few bolts), resulting in a slight change of the relation between the lower leg angle and motor angle (the curve of Fig. 5 would be shifted a little). However, it was very hard to tune it such that the curve would exactly go through the point $(\alpha, \varphi)=(0,0)$. Moreover, temperature changes, shocks or falls of the robot could de-tune the system easily. The actual relation could be such that when $\alpha=0$ then $\varphi<0$, i.e., the curve is shifted somewhat to the left. The end stop in the knee would not allow the lower leg to have a negative angle, implying that the motor arm could never pass the singularity. Oppositely, the relation could be such that when $\alpha=0$ then $\varphi>0$, i.e., the curve is shifted somewhat to the right. In this case, the motor arm can move through its whole stroke. Near the singularity, the lower leg exerts a large force on the motor arm, perpendicularly to its moving direction. This force creates a lot (theoretically: infinity) of friction in the rotational joints. Because of this friction, the motor arm would stop moving, again not passing its singularity.

From Fig. 5 it can be seen that when some external torque on the lower leg would force it to be fully extended, the motor arm will be pushed towards the singularity, diminishing the robustness of the lock (the motor arm could easily go past the singularity, thereby unlocking). The VCA could of course be used to counteract this movement, but this consumes power, which is unwanted.

In order to solve all these problems, we deliberately created play into the system: we made the hole on joint $J_{l}$ approximately half a millimeter larger than the axis. The result is that the motor arm and lower leg are now able to move a little, independently from each other. Fig. 8 shows all possible combinations of motor arm and lower leg angles. From the figure, a few interesting observations can be made. There is still a single point (point A) where the bending moment of the knee self-locks. Due to the play it has moved a little bit (the leg is more flexed), but practically this cannot be noticed. The real benefit is in the line B1B2. This line shows that, when the leg is fully extended $(\varphi=0$, against the leg's end stop), the motor arm can move freely all the way from its end-stop (B1) to a point past the singularity 


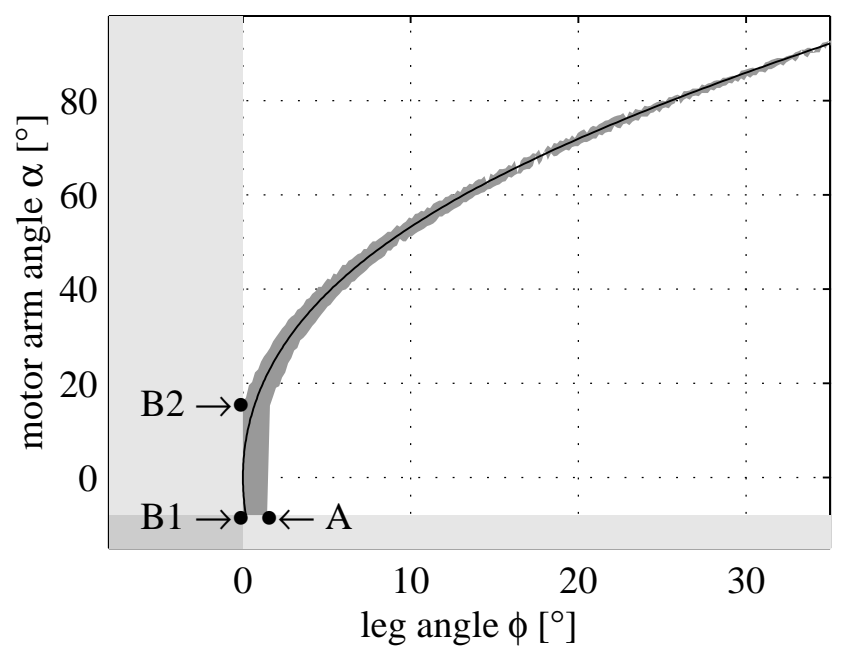

Fig. 8. Measurement of the relation between the motor arm angle and lower leg angle - Due to the mechanical play, there is no exact coupling between the two. The black line indicates the theoretical no-play situation. Point A: self-locking point when there is a bending moment on the lower leg. Line B1B2: The range of motor arm angles 'compatible' with a fully extended leg.

(B2): as long as $\varphi=0$, the motor arm movement is fully uncoupled from the leg for a quite large stroke.

Fine-tuning (or accidental de-tuning), may still shift the curve (which is now actually a region) of Fig. 8, but now there is a tuning margin for which the point $(\alpha, \varphi)=(0,0)$ is within the region. Hence, the motor arm is always able to pass the singularity when the leg is fully extended. Moreover, when the leg is fully extended by an external torque, all torque is counteracted by the leg's end stop; the leg does not exert any force on the motor arm anymore. This way, we have got rid of the friction issues and the motor arm will not be pushed back towards the singularity. The motor arm can be held against its end stop easily by a small amount of power on the VCA, greatly reducing the chances of accidental unlocking.

This means that by creating play in the system, we made it more robust and far less sensitive to tuning. A super-simple solution with great benefit!

\section{CONCLUSIONS AND FUTURE WORK}

We have designed, implemented and tested a new knee locking mechanism for the energy efficiently walking robot Dribbel. It uses a four-bar mechanism and a mechanical singularity for locking the knee in extended position. Once extended, the knee remains locked without energy consumption, while un-locking only costs a small amount of energy. We used suction cups for passive de-bouncing of the system, which has been proven to be an effective method. A simple controller was implemented that is locally executed by the knee-module. The tests showed that the knee locks function well, that the energy consumption indeed has decreased and the robot walks robustly.

Future work will focus on improving the control algorithm. Firstly, the energy consumption could be further decreased by actively monitoring the exact movement of the motor arm, and only activate the VCA if strictly necessary. For example, when locking, if the motor arm is already moving towards the end stop with enough velocity, it is not necessary to help it by turning on the VCA. Secondly, the VCA could be used for active de-bouncing. If, when locking, the motor arm goes really fast, the VCA could be used to decelerate it, reducing the chance of a rebound. Both enhancements require more accurate measurement of the motor arm angle $\alpha$, which will be provided by a more accurate sensor.

\section{REFERENCES}

[1] E. Dertien, "Realisation of an energy-efficient walking robot," MSc thesis 022CE2005, University of Twente, June 2005.

[2] M. Franken, "Ankle actuation for planar bipedal robots," MSc thesis 012CE2007, University of Twente, Apr. 2007.

[3] R. Reilink, "Knee locking systems for a walking robot," BSc thesis 012CE2009, University of Twente, July 2009.

[4] D. Borgerink, "Design and implementation of a knee mechanism for walking robots," BSc thesis 027CE2008, University of Twente, Dec. 2008.

[5] T. McGeer, "Passive dynamic biped catalogue, 1991," in The 2nd International Symposium on Experimental Robotics II. London, UK: Springer-Verlag, 1993, pp. 463-490.

[6] M. Wisse and J. V. Frankenhuyzen, "Design and construction of MIKE; a 2D autonomous biped based on passive dynamic walking," in Proceedings of International Symposium of Adaptive Motion and Animals and Machines, 2003.

[7] S. Collins and A. Ruina, "A bipedal walking robot with efficient and human-like gait," in Proceedings of IEEE International Conference on Robotics and Automation, 2005, pp. 1995-2000.

[8] A. Baines, "Knee design for a bipedal walking robot based on a passive-dynamic walker," Massachusetts Institute of Technology, Tech. Rep., June 2005.

[9] A. Reynolds, "Design and control of a clutch for a minimally-actuated biped based on the passive-dynamic simple walker," Massachusetts Institute of Technology," BSc report, June 2006.

[10] K. Trifonov and S. Hashimoto, "Active knee-lock release for passivedynamic walking machines," Proceedings of IEEE International Conference on Robotics and Biomimetics, pp. 958 - 963, 2007.

[11] R. Z. Marlow, "Artificial knee with improved stable link-type knee joint," U.S. Patent No. 4911709, Mar. 1990.

[12] J. H. Townsend, "Multiaxis controlled motion knee brace with a four bar joint and method for producing same," U.S. Patent No. 5330418, July 1994.

[13] S. Kramer, "Knee joint mechanism for knee disarticulation prosthesis," U.S. Patent no. 5746774, 1998.

[14] C. Radcliffe, "Knee brace with pivot lock," U.S. Patent No. 5168865, Dec. 1992.

[15] J. H. Townsend, "Orthopedic knee brace joint assembly having a trigger locking mechanism," U.S. Patent No. 6500139, Dec. 2002.

[16] J. Hieronymus, "Edge folding device having self-locking mechanism," U.S. Patent No. 6588341, July 2003.

[17] M. Brian, "HowStuffWorks - How hard disks work," http://computer.howstuffworks.com/hard-disk.htm.

[18] C. D. Quillfeldt, "Improvement in bottle-stoppers," U.S. Patent No. 158406, Jan. 1875.

[19] M. Wisse, A. L. Schwab, R. Q. Van Der Linde, and F. C. T. van der Helm, "How to keep from falling forward: elementary swing leg action for passive dynamic walkers," IEEE Transactions on Robotics, vol. 21, no. 3, pp. 393 - 401, June 2005. 